IZABELA MARTINA RAMOS RIBEIRO DE TOLEDO

\title{
ESTUDOS ANATOMO-FUNCIONAIS DE VIAS INTEGRATIVAS HIPOTALÂMICAS E BULBARES ENVOLVIDAS NO CONTROLE DO SISTEMA NERVOSO AUTÔNOMO DECORRENTES DA AÇÃO CENTRAL DA INSULINA
}

\author{
Tese apresentada ao Programa de Pós- \\ Graduação em Fisiologia Humana do \\ Instituto de Ciências Biomédicas da \\ Universidade se São Paulo, para obtenção \\ do Título de Doutora em Ciências. \\ Área de concentração: Fisiologia Humana \\ Orientador: Prof. Dr. Vagner Roberto Antunes
}

SÃO PAULO

2016 


\section{RESUMO}

Ribeiro IMR. Estudos anatomo-funcionais de vias integrativas hipotalâmicas e bulbares envolvidas no controle do sistema nervoso autônomo decorrentes da ação central da insulina. [Tese (Doutorado em Fisiologia Humana)]. São Paulo: Instituto de Ciências Biomédicas, Universidade de São Paulo, 2016.

A glicose é o mais importante substrato energético utilizado pela maioria das células corporais e portanto, sua concentração plasmática deve ser finamente controlada a fim de evitar variações na sua disponibilidade. 0 fígado desempenha uma função essencial no controle da produção de glicose, uma vez que é capaz de sintetizar, armazenar e liberar glicose para a circulação em situações de alimentação e repouso. Hormônios como insulina e catecolaminas influenciam a PHG mas pouco se conhece acerca da ação central de doses fisiológicas de insulina na modulação da PHG através do SNA em ratos não anestesiados especialmente em SHR que tem como característica resistência à insulina dentre outras disfunções metabólicas. Estudos anteriores do nosso laboratório mostraram uma diminuição de $27 \%$ na glicemia medida diretamente na veia hepática (CGVH) de ratos Wistar já ao 10 minutos após a injeção ICV de insulina $(100 \mathrm{nU} / \mathrm{mL})$ sem alterações significantes (3\%) em SHR (Ribeiro et al., 2015). O pré-tratamento com metil-atropina bloqueou totalmente a redução CGVH, enquanto que o pré-tratamento com propranolol e fentolamina induziu uma diminuição de $8 \%$ em CGVH após injeção ICV de insulina em ratos Wistar. Em um grupo separados de animais buscamos avaliar os efeitos de infusões ICA de insulina sobre a atividade do SVN e do ramo esplâncnico do LSSN. A injeção de insulina ICA promoveu um aumento significativo na atividade SVN em ratos Wistar $(12 \pm 2 \%)$, enquanto nenhuma diferença significativa foi observada na atividade do LSSN $(-6 \pm 3 \%)$. Além disso, esta mesma abordagem experimental não causou qualquer alteração no SVN e LSSN $(-2 \pm 2 \%$ e $2 \pm 3 \%$, respectivamente) em SHR após a injeção ICA de insulina. Ainda, um novo grupo experimental mostrou que a insulina injetada no VL promoveu um aumento significativo da imunorreatividade para a proteína FOS no PVN quando comparado ao grupo controle $(412 \pm 44$ cél/ $\mathrm{mm}^{2}$ e $131 \pm 57 \mathrm{cél} / \mathrm{mm}^{2}$, respectivamente). Além disso, por meio da técnica de depósito do traçador retrógrado FluoroGold $\AA$ no $\mathrm{DMV}$, demonstramos projeções diretas para este núcleo, provindas do PVN. Em conjunto, estes resultados mostram em animais não anestesiados a importância do sistema nervoso parassimpático no controle da CGVH pela insulina central em animais Wistar, o qual não é efetivo em SHR. Além disso, a ação central da insulina envolve a participação do PVN, um núcleo hipotalâmico essencial que mantém projeções com o núcleo motor dorsal e que em conjunto podem determinar a integração do SNA na ação central da insulina. Em um novo grupo experimental, procuramos avaliar os efeitos do treinamento físico aeróbio na redução da glicemia em resposta à insulina injetada centralmente e mostramos que após 4 semanas, os animais submetidos ao treinamento físico tiveram uma queda de $24 \%(23 \pm 5 \mathrm{mg} / \mathrm{dL})$ na glicemia, quando comparados aos seus controles sedentários $(4 \pm 3 \mathrm{mg} / \mathrm{dL})$. No entanto, não observamos variações na concentração plasmática de insulina nos animais treinados $(0.29 \pm 0.05 \mathrm{ng} / \mathrm{ml})$ quando comparados aos seus controles sedentários $(0.25 \pm 0.03 \mathrm{ng} /$ $\mathrm{ml})$, bem como na sensibilidade periférica à esse hormônio $(2.9 \pm 0,1 \% / \mathrm{min}$ treinados $x 2.6 \pm 0,3 \% /$ min sedentários). 
Palavras-chave: Ação central da insulina. Sistema nervoso autônomo. SHR. Exercício físico 


\begin{abstract}
Ribeiro IMR. Estudos anatomo-funcionais de vias integrativas hipotalâmicas e bulbares envolvidas no controle do sistema nervoso autônomo decorrentes da ação central da insulina. [Ph. D. thesis (Human Physiology)] - São Paulo: Instituto de Ciências Biomédicas, Universidade de São Paulo, 2016.
\end{abstract}

Glucose is the main energy substrate used by most body cells and therefore its plasma concentration must be finely controlled in order to avoid variations in availability. The liver plays a key role in the control of glucose production, since it is able to synthesize, store and release glucose into the circulation at rest and feed conditions. Hormones such as insulin and catecholamines influence PHG but little is known about the physiological centrally acting insulin doses in modulating PHG through SNA rats unanesthetized especially in SHR has the characteristic of insulin resistance among other metabolic disorders. Previous studies from our laboratory showed a $27 \%$ decrease in blood glucose measured directly into the hepatic vein (CGVH) Wistar rats already at 10 minutes after insulin ICV injection $(100 \mathrm{nU} / \mathrm{ml})$ without significant changes (3\%) in SHR ( Ribeiro et al., 2015). The pre-treatment with atropine methyl-CGVH completely blocked the reduction, whereas pretreatment with propranolol and phentolamine induced a decrease of $8 \%$ by $\mathrm{CGVH}$ insulin after ICV injection in Wistar rats. In a separate group of animals we sought to evaluate the effects of insulin infusions ICA on the activity of SVN and splanchnic branch Issn. ICA insulin injection caused a significant increase in SVN activity in Wistar rats (12 $\pm 2 \%)$, while no significant difference was observed in the activity of Issn $(-6 \pm 3 \%)$. Moreover, this same experimental approach did not cause any change in the SVN and Issn (-2 $\pm 2 \%$ and $2 \pm 3 \%$, respectively) in SHR after insulin injection ICA. Still, a new experimental group showed that insulin injected into the VL caused a significant increase in immunoreactivity for FOS protein in the PVN compared to the control group (412 \pm 44 cells / mm2 and $131 \pm 57$ cells / mm2, respectively). Furthermore, by depositing tracer technique retrograde FluoroGold ${ }^{\circledR}$ in DMV demonstrated direct projections for this core, emanating from the PVN. Together, these results show unanesthetized animals the significance of the parasympathetic nervous system in the central control CGVH Wistar insulin, which is not effective in SHR. Furthermore, the central insulin action involves the participation of PVN, an essential hypothalamic nucleus which retains projections with the dorsal motor nucleus and which together may provide the integration of central autonomic nervous system in the action of insulin. In a new experimental group, we evaluate the effects of physical training in reducing blood glucose in response to insulin injected centrally and showed that after 4 weeks, the animals submitted to the physical training showed a decrease of $24 \%$ (23 $\pm 5 \mathrm{mg} / \mathrm{dL}$ ) glycemia, when compared to their sedentary controls ( $4 \pm 3 \mathrm{mg} / \mathrm{dL}$ ). However, we observed no changes in plasma insulin concentration in trained animals $(00: 29 \pm 0.05 \mathrm{ng} / \mathrm{ml})$ when compared to their sedentary controls $(12: 25 \pm 0.03 \mathrm{ng} /$ $\mathrm{ml})$ and in peripheral sensitivity to this hormone $(2.9 \pm 0,1 \% / \mathrm{min}$ - treined $\times 2.6 \pm$ $0.3 \% /$ min- control).

Keywords: Central action of insulin. Autonomic nervous system. SHR. Physical exercise. 
A glicose é um dos mais importantes carboidratos utilizados como substrato energético pela maioria das células em diversos organismos, desde as bactérias até os humanos por meio de respiração aeróbica, anaeróbica e fermentação. resentando em sua estrutura 6 átomos de carbono, é classificada como uma hexose, uma sub-categoria de monossacarídeo importante na a manutenção da função de diversos tecidos corporais. Dentre eles, destaca-se o tecido nervoso, que requer um contínuo suprimento de glicose para manutenção de sua alta demanda energética bem como para a manutenção de sua homeostase metabólica. Um exemplo disso é que uma grande parte da energia provida pela glicose é usada somente para a manutenção do potencial de repouso de membrana dos neurônios (Verbene et al., 2014).

Dada essa importância para a sobrevivência da maioria das células, é certo que a concentração plasmática de glicose deve ser finamente controlada a fim de evitar até mesmo mínimas variações na sua disponibilidade (Pilkis , Granner, 1993). Em situaçõwa patológicas, como por exemplo, no diabetes mellitus tipo I que se caracteriza por uma alta concentração circulante de glicose (hiperglicemia) pode ter um grande impacto na saúde do indivíduo, incluindo doenças cardiovasculares, retinopatia, neuropatia e nefropatia. O oposto disso, a hipoglicemia, pode levar a uma série de alterações como convulsões, perda de consciência e até mesmo a morte. Assim, afim de restaurar a normoglicemia, são ativados uma série de mecanismos conhecidos como "contra regulatórios" (Verbene et al., 2014).

Neste sentido, diversos mecanismos se responsabilizam pela manutenção dos níveis glicêmicos adequados às demandas energéticas de cada tecido em particular.

O fígado possui uma função essencial na manutenção da homeostase glicêmica por controlar a produção de glicose, uma vez que é capaz de sintetizar, armazenar e liberar glicose para a circulação em situações de alimentação e jejum, além de controlar a liberação desse substrato para a manutenção de sua própria demanda energética (Jones, 2016; Nordlie et al., 1999; Perseghin et al., 1997).

No entanto, a refinada manutenção de níveis glicêmicos adequados para a demanda metabólica do organismo requer a ação conjunta de diversos mecanismos de controle, os quais envolvem além do já citado papel do fígado, mecanismos hormonais de dois grupos de hormônios com efeitos antagônicos (catabólicos 
anabólicos) que se revezam na regulação da glicemia dependendo do estado alimentar do indivíduo.

Dentre os hormônios catabólicos destaca-se o glucagon que é produzido pelo pâncreas e responsável pelo aumento nos níveis glicêmicos em situações como privação alimentar. Já na categoria de hormônios anabólicos destaca-se a insulina que exerce uma função de destaque na redução dos níveis glicêmicos. A insulina é sintetizada no pâncreas e sua ação é controlar de maneira direta a captação de glicose por tecidos específicos, além de atuar na sinalização hepática no controle da captação e liberação de glicose para a circulação em situações específicas regulando desta maneira a disponibilidade de glicose (Aronoff et al., 2004).

A secreção de insulina a partir das vesículas que contém este hormônio no interior das células beta pancreáticas é estimulada por substratos energéticos metabolizáveis por esta célula, sendo a glicose o estímulo mais importante para essa secreção.

Com o aumento na sua concentração plasmática, a glicose é transportada para o interior da célula beta por uma proteína integral de membrana (GLUT2). Essa proteína possui um elevado $\mathrm{Km}$ (entre 15 e $20 \mathrm{mmol} / \mathrm{L}$ ) garantido que não seja saturável em concentrações fisiológicas de glicose, e um Vmax muito elevado, permitindo um aumento rápido no transporte da glicose quando a glicemia se eleva.

No interior da célula pancreática o metabolismo da glicose gera trifosfato de adenosina (ATP) e assim a fração ATP/ADP aumenta no citoplasma provocando o fechamento de canais de potássio com consequente despolarização da membrana e abertura de canais de cálcio sensíveis à voltagem. $O$ influxo de cálcio resulta em uma despolarização suplementar da membrana desencadeando o processo de exocitose das vesículas contendo insulina em seu interior (Haber et al., 2001).

Parte do processo de secreção de insulina pela célula beta pancreática está ilustrada na figura 1. 


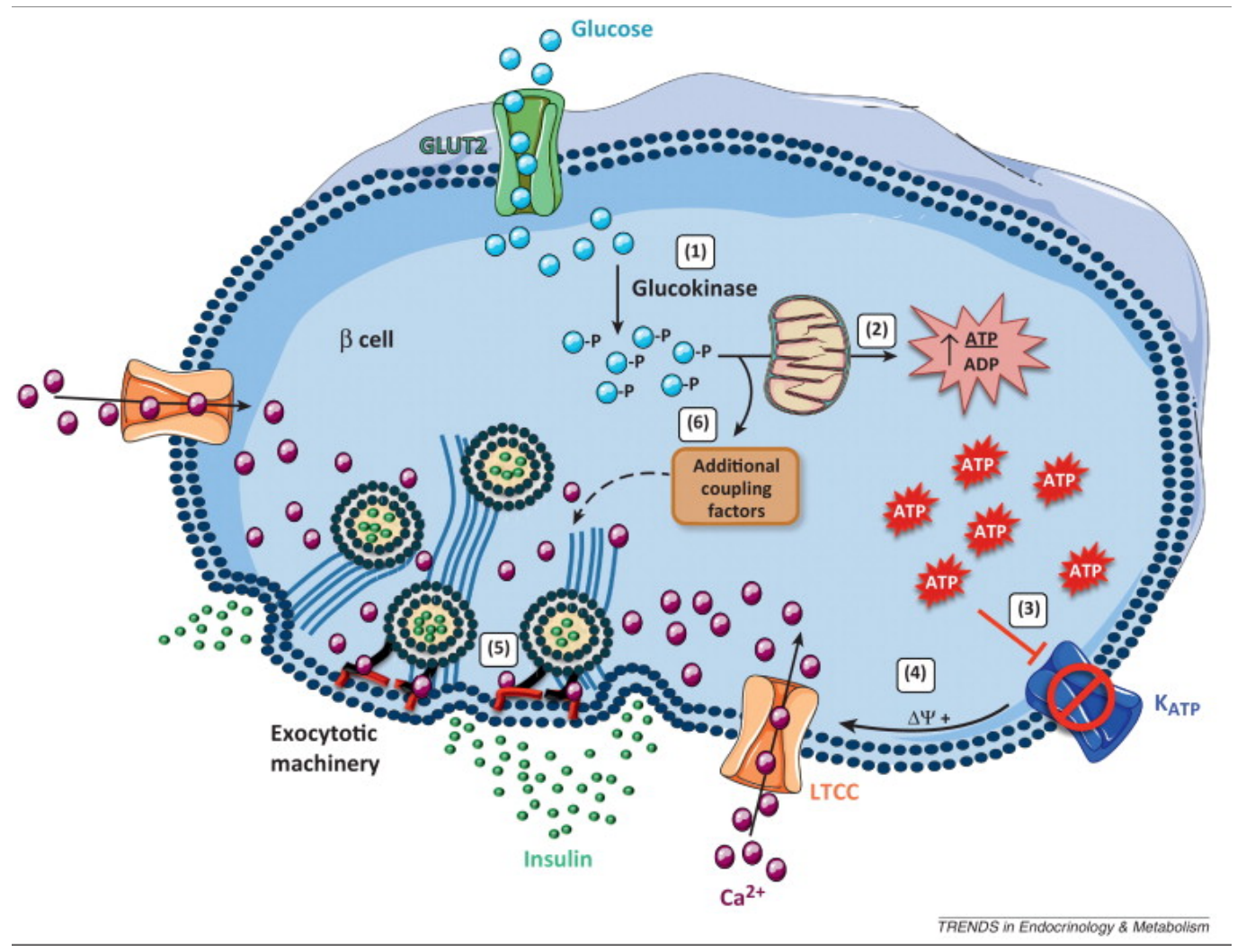

Figura 1- Secreção de insulina pela célula beta-pancreática Fonte: Mancini e Poitoit (2013)

A molécula "madura" de insulina, consiste em duas cadeias peptídicas, a cadeia $A$, com 21 aminoácidos e a cadeia $B$, com 30 aminoácidos ligadas entre si por duas pontes dissulfeto. Sua ação na célula, inicia-se pela ligação do hormônio ao receptor de membrana, uma proteína heterotetramérica, constituídas por duas subunidades alfa (extracelulares), onde há um sítio de ligação para a insulina, e duas subunidades beta (transmembranas) responsáveis pela transmissão do sinal, com atividade tirosina cinase (Kasuga et al., 1982). Agindo como um doador de fosfatos, o ATP promove a fosforilação do receptor em resíduos de serina. Sendo assim, a insulina induz a autofosforilação do receptor, aumentando a sua capacidade de fosforilar um ou mais substratos proteicos intracelulares. A fosforilação de seus substratos dá início a uma série de eventos incluindo a cascata de reações de fosforilação e defosforilação que regula os seus efeitos metabólicos e de crescimento (Haber et al., 2001). O resumo dos eventos que ocorrem após a ligação da insulina em seu receptor estão ilustrados na figura 2 a seguir. 


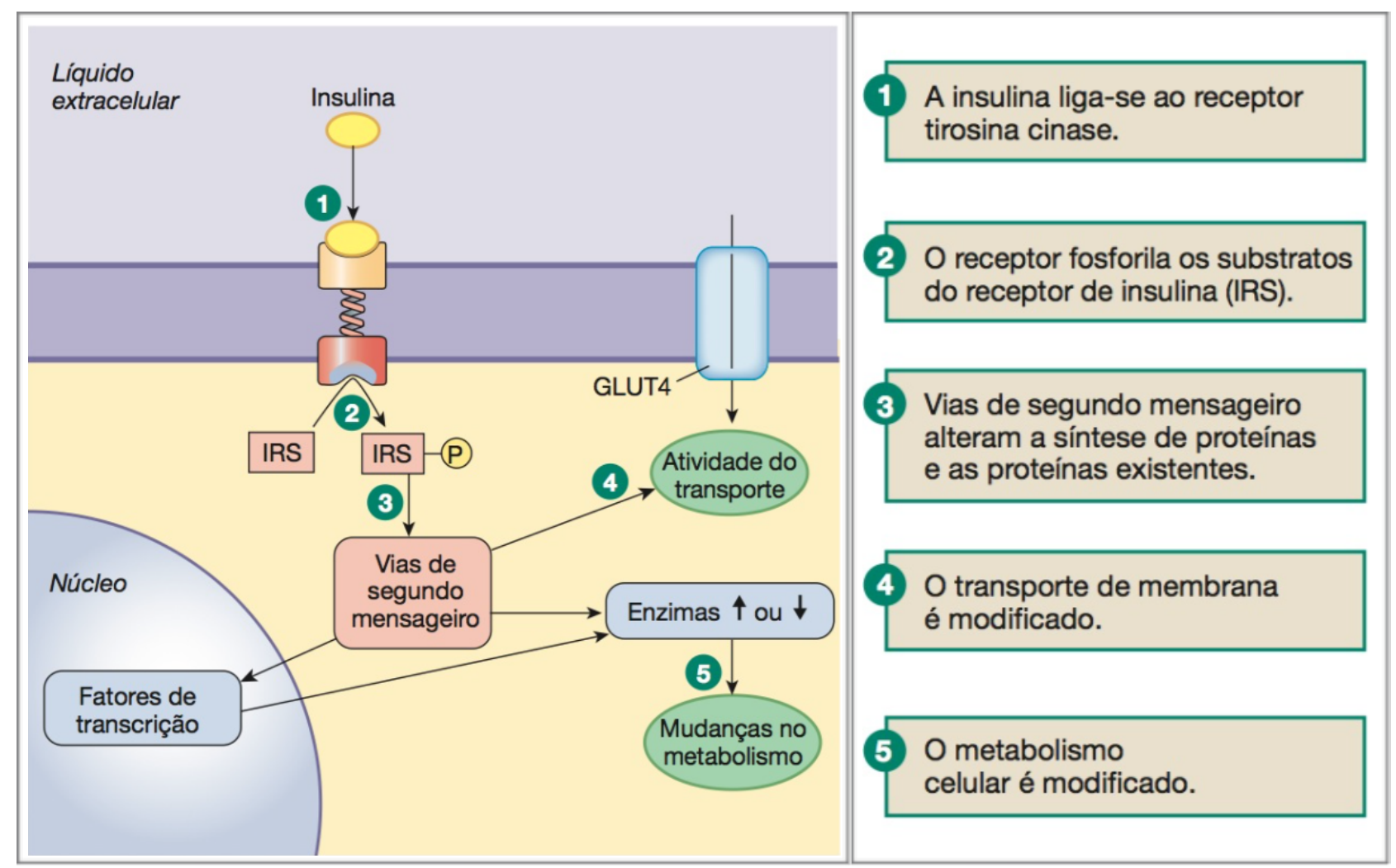

Figura 2- Vias de sinalização da insulina.

Fonte: Silverthorn (2010)

Como já mencionado, a insulina desempenha uma função ubíqua tendo seus receptores distribuídos por praticamente todas as células corporais e assim modula a função de diversos órgãos. Seus efeitos mais conhecidos são demonstrados i) nas células endoteliais, onde estimula vasodilatação e recrutamento capilar, ii) no cardiomiócito, sendo responsável pela função e metabolismo normais destas células, iii) atuando no sistema imune regula a invasão de macrófagos do tecido adiposo branco possuindo uma ação antiinflamatória, iv) nos adipócitos possui uma importante ação antilipolítica, v) estimula a captação de glicose no músculo esquelético, vi) agindo no fígado suprime a gliconeogênese e glicogenólise e aumenta a síntese de glicogênio e vii) nas células beta-pancreáticas atua como reguladora de sua própria secreção bem como age na manutenção da quantidade de células beta. (Rask-Madsen, Kahn, 2012). 
No sistema nervoso central (SNC) a insulina desempenha uma série de funções, tais como aprendizado, memória, assim como na regulação da homeostase energética (Plum et al., 2006). De particular interesse a insulina exerce uma importante ação no hipotálamo suprimindo a liberação de glicose pelo fígado como já demonstrado pelo nosso laboratório (Ribeiro et al., 2015).

Tais ações da insulina bem como o que acontece no metabolismo durante uma situação de resistência à esse hormônio e consequente perda de sua atividade estão resumidos na figura 3.

A função da insulina no controle da glicemia também passa pela ação coordenada do sistema nervoso autônomo (SNA) que influencia diretamente os níveis circulantes de glicose (Perseghin et al., 1997), sendo esse, o foco de investigação no presente trabalho.

O eixo de vias neurais são importantes para a comunicação entre o fígado e outros órgãos no controle do metabolismo corporal. Tipicamente, os aferentes vagais do trato gastrointestinal (TGI) levam informações ao cérebro sobre o influxo de nutrientes enquanto regiões sensoriais centrais coordenam o gasto e a reserva energética de todo o corpo. Neste sentido, o crosstalk entre TGI e cérebro representa uma maquinaria coordenada conectando fatores ambientais e metabolismo da glicose (Zhang et al., 2016). 




Figura 3- $\quad$ Fisiologia da sinalização da insulina.

Fonte: Rask-Madsen e Kahn (2012)

Evidências acumuladas nos últimos anos, demonstram através de técnicas como clamp euglicêmico-hiperinsulinêmico, a ação sistêmica periférica da insulina na modulação da atividade do sistema nervoso autônomo de ratos (Morgan et al., 1993) e humanos (Berne et al., 1992) mostrando que elevados níveis plasmáticos de insulina são capazes de promover um aumento na atividade simpática de maneira não uniforme para territórios específicos. 
Além de suas ações periféricas, já no início da década de 1980, estudos mostraram que o cérebro (mais precisamente o hipotálamo) é um órgão sensível à ação da insulina (Szabo et al., 1983; Obici et al., 2002; Pocai et al., 2005).

Szabo et al. (1975) demonstraram a influência da insulina agindo centralmente (injetada na artéria carótida) na modulação da glicemia hepática de animais anestesiados. Outros trabalhos abordando esse tema, evidenciaram que a insulina agindo especificamente no hipotálamo (microinjeção no terceiro ventrículo) promoveu queda na PHG (Obici et al., 2002; Pocai et al., 2005) defendendo a teoria de que a gliconeogênese hepática seja afetada negativamente pela ação central da insulina por ação em núcleos hipotalâmicos específicos.

Abordando este tema, estudos anteriores do nosso laboratório Ribeiro et al. (2015) conduzidos em ratos não anestesiados, mostraram uma importante queda na concentração de glicose na veia hepática (CGVH) de ratos Wistar, mas não em SHR, após injeção ICV de baixas doses insulina. Ainda, foi demonstrado o importante papel da alça parassimpática do SNA na redução da glicemia em resposta à insulina injetada centralmente, por meio da prévia administração do antagonista de receptores muscarínicos colinérgicos, metil-atropina, que bloqueou totalmente a redução da CGVH. Estes resultados vão ao encontro de estudos prévios conduzidos por Szabo et al. (1983) que demonstraram que a vagotomia impede a supressão da produção hepática de glicose (PHG) induzida pela injeção central de insulina diretamente no hipotálamo ventromedial de animais anestesiados.

Além disso, estudos e Ribeiro et al. (2015) também demonstraram uma menor, mas significativa influência da alça simpática do SNA evidenciado por uma diminuição na resposta de queda da glicemia em decorrência da ação central de insulina em animais cujo antagonismo periférico de receptores alfa e beta adrenérgicos com fentolamina e propranolol, respectivamente, foi administrado previamente à insulina. Estes resultados corroboram com dados prévios da literatura que demonstram que injeção de insulina no terceiro ventrículo modula a atividade simpática de maneira não uniforme para territórios distintos sem alterar parâmetros cardiovasculares como pressão arterial e frequência cardíaca, ou seja, agindo de maneira específica e não global (Muntzel et al.,1994,1995). Desta forma, fica clara a 
influência exercida pela insulina agindo centralmente na modulação do SNA no controle da glicemia.

Como visto, a ação da insulina agindo tanto periférica quanto centralmente já foi alvo de inúmeras pesquisas. No entanto, um assunto que merece maior atenção é a ação e efeito de baixas doses (dentro da faixa fisiológica) de insulina na modulação do SNA para territórios específicos, como fígado, em animais não anestesiados, já que os anestésicos de maneira geral, exercem uma grande influência no balanço simpato-vagal. Ainda, se faz necessário o conhecimento mais aprofundados das vias centrais de ação da insulina bem como as conexão existente entre os núcleos hipotalâmicos e bulbares envolvidos no controle do SNA e finalmente da glicemia pela insulina injetada centralmente.

Devido a essa importante função da insulina na modulação da atividade autonômica sabe-se que situações de resistência à insulina e hiperinsulinemia estão intimamente relacionadas à hipertensão arterial já que há muitos efeitos da insulina nos rins, SNS e sistema cardiovascular (Brands et al., 1992). Neste sentido, estudos demonstram que anormalidades no metabolismo da glicose e da insulina são comuns não só em pacientes hipertensos como em parentes (normotensos) de primeiro grau desses indivíduos, o que não é encontrado em indivíduos com quadro de hipertensão secundária. Ainda, nesses casos, as anormalidades do metabolismo da glicose e da insulina, não necessariamente melhoram com a queda da PA a partir do uso de fármacos anti-hipertensivos (Reaven et al., 1996).

Neste sentido, diversos estudos vem demonstrando a eficácia do exercício físico como terapia adjuvante no tratamento da hipertensão arterial e diabetes mellitus não insulino-dependente (Bogardus et al., 1984; Cornelisses , Smart, 2013; Dela et al., 1994; Hamberg et al., 2000; Krotkiewski et al., 1985).

A Hipócrates é atribuído o pensamento de que "Somente a comida não manterá um homem sadio, ele também precisa fazer exercícios. Comida e exercício, embora sejam qualidades opostas, trabalhando juntas, produzem saúde". Essas palavras, mostram que a atividade física aliada a uma dieta equilibrada é uma ideia reconhecida há muito tempo como uma importante ferramenta na manutenção da saúde (Jones, Withington, $1931^{1}$ apud Short, 2013).

De fato, há de se reconhecer a importância de uma terapia não farmacológica, tal como o exercício físico, no tratamento de complicações tão 
comuns encontradas na população bem como para a promoção da saúde. Portanto, postulamos a tese de que o exercício físico pode se mostrar eficaz na modulação da redução da glicemia em resposta à insulina injetada centralmente nos ratos SHR que se mostraram resistentes a esse efeito, modulando não só a resposta à insulina como também alterando a concentração plasmática de insulina e sensibilidade à insulina destes animais. 
7 CONCLUSÃO 
O treinamento físico aeróbio é capaz de restabelecer os efeitos centrais da insulina na sua função em modular a glicemia de SHR e a ação deste hormônio no SNC depende da ativação de uma conexão entre um núcleo hipotalâmico (PVN) e bulbar (DMV) que compõe uma via integrativa do sistema nervoso autônomo parassimpático. 
REFERÊNCIAS* 
Antunes VR, Yao ST, Pickering AE, Murphy D, Paton JF. A spinal vasopressinergic mechanism mediates hyperosmolality-induced sympathoexcitation. J Physiol. 2006;576:569-583.

Ao Y, Wu S, Go VL, Toy N, Yang H. Maintaining euglycemia prevents insulin-induced Fos expression in brain autonomic regulatory circuits. Pancreas. 2005; 31:142-147.

Aronoff SL, Berkowitz K, Shreiner RN, Want L. Glucose metabolism and regulation: beyond insulin and glucagon. Diabetes Spectrum. 2004;17:183-190.

Berne C, Fagius J, Pollare T, Hjemdahl P. The sympathetic response to euglycaemic hyperinsulinaemia. Evidence from microelectrode nerve recordings in healthy subjects. Diabetologia. 1992;35:873-9.

Borgadus C, Ravussin E, Robbins DC, Wolfe RR, Horton ES, Sims EAH. Effects of Physical Training and Diet Therapy on Carbohydrate Metabolism in Patients with Glucose Intolerance and Non-insulin-dependent Diabetes Mellitus. Diabetes. 1984;4: 311-318.

Cornelissen VA, Smart NA. Exercise Training for Blood Pressure: A Systematic Review and Meta- analysis. J Am Heart Assoc. 2013;2:1-9.

Dela F, Ploug T, Handberg A, Petersen LN, Larsen JJ, Mikines KJ, Galbo H. Physical Training Increases Muscle GLUT4 Protein and mRNA in Patients With NIDDM. Diabetes. 1994;7:862-865.

Dragunow M. Faull R. The use of FOS as a metabolic marker in neuronal pathway tracing. J Neurosc Methods. 1989;3:261-5.

Hagberg JM, Park JJ, Brown MD. The role of exercise training in the treatment of hypertension: an update. Sports Med. 2000;3:193-206.

Herrera DG, Robertson HA. Activation of FOS in the brain. Progr in Neurobiol. 1996;50:83-107.

Hulman S, Falkner B, Freyvogel N. Insulin resistance in the conscious spontaneously hypertensive rat: euglycemic hyperinsulinemic clamp study. Metabolism. 1993;42:14-18.

* De acordo com: International Comittee of Medical Journal Editors.

[Internet] Uniform requirements for manuscripts submitted to biomedical journals [2011 Jul 15]. Avaliable from: http:// www.nlm.nih.gov/bsd/uniform_requirements.htlm 
Ichige MHA, Santos CR, Jordão CP, Ceroni A, Negrão CE, Michelini LC. Exercise training preserves vagal preganglionic neurones and restores parasympathetic tonus in heart failure. J Physiol. 2016. In Press.

Image-Pro Plus. On line: Image-Pro Plus. Version 7.0 [cited 2016 Out 26] Available from: http://www.mediacy.com/imageproplus.

Jensen KJ, Alpini G, Glaser S. Hepatic Nervous System and Neurobiology of the Liver. Compr Physiol. 2013;3:655.

Jones JG. Hepatic glucose and lipid metabolism. Diabetologia. 2016;59:1098-1103.

Krotkiewski M, Lönnroth P, Mandroukas K, Wroblewski Z, Rebuffé-Scrive M, Holm G, Smith U, Björntorp P. The effects of physical training on insulin secretion and effectiveness and on glucose metabolism in obesity and Type 2 (non-insulindependent) diabetes mellitus. Diabetologia. 1985;28:881-890.

LabChart Pro. On line: LabChartPro. Version 8.1.5. [cited 2016 Out 26] Available from: https://www.adinstruments.com/support/software\#labchart-downloads.

Laughton WB, Powley TL. Localization of efferent function in the dorsal motor nucleus of the vagus. Am J Physiol Regul Integr Comp Physiol. 1987;1:13-25.

Luiten PG, ter Horst GJ, Karst H, Steffens AB. The course of paraventricular hypothalamic efferents to autonomic atructures in medulla and spinal cord. Brain Res. 1985;329:374-378.

Mancini AD, Poitout $\mathrm{V}$. The fatty acid receptor FFA1/GPR40 a decade later: how much do we know? Trends Endocrinol Metabol. 2013;24:398-407.

Masson GS, Costa TSR, Yshii L, Fernandes DC, Soares, PPS, Laurindo FR, Scavone C, Michelini LC. Time-Dependent Effects of Training on Cardiovascular Control in Spontaneously Hypertensive Rats: Role for Brain Oxidative Stress and Inflammation and Baroreflex Sensitivity. Plos One. 2014;9:1-10.

Morgan DA, Balon TW, Ginsberg BH Mark AL. Nonuniform regional sympathetic nerve responses to hyperinsulinemia in rats. Am. J. Physiol. 1993;264:423-427.

Muntzel MA, Anderson EA, Johnson AK, Mark AL. Mechanisms of insulin action on sympathetic nerve activity. Clin Exp Hypertens. 1995;17:39-50.

Muntzel MS, Morgan DA, Mark A, Johnson AK. Intracerebroventricular insulin produces nonuniform regional increases in sympathetic nerve activity. Am J Physiol. 1994;267:1350-1355.

Nordlie RC, Foster JD. Regulation of glucose production by the liver. Annu. Rev. Nutr. 1999;19:379-406. 
Obici S, Zhang BB, Karkanias G, Rosseti. L. Hypothalamic insulin signaling is required for inhibition of glucose production. Nat. Med. 2002;8:1376-1382.

Paton JFR. A working heart-brainstem preparation of the mouse. Journal of Neuroscience Methods. 1996;65:63-68.

Paxinos G, Watson C. The rat brain in stereotaxic coordinates. 7 ed. New York: Academic Press, 2013.

Pearse W. The cardiovascular autonomic nervous system and anesthesia. S Afr. J. Anesthe. Anal. 2002;8:8-24.

Perseghin G, Regalia E, Battezzati A, Vergani S, Pulvirenti A, Terruzzi I, Baratti D, Bozzetti F, Mazzaferro V, Luzi L. Regulation of glucose homeostasis in humans with denervated livers. J. Clin. Invest. 1997;100:931-941.

Pilkis SJ, Granner DK. Molecular physiology of the regulation of the hepatic gluconeogenesis and glycolysis. Annu. Rev. Physiol. 1992;54:885-909.

Plum L, Belgardt BF, Brüning JC. Central insulin action in energy and glucose homeostasis. J Clin Invest. 2006;7:1761-1766.

Pocai A, Lam TK, Gutierrez-Juarez R, Obici S, Schwartz GJ, Bryan J. Hypothalamic K(ATP) channels control hepatic glucose production. Nature. 2005;434:1026-1031.

Prism. On line: GraphPad. Version 7. [cited 2016 Out 26] Available from: http:// www.graphpad.com

Rask-Madsen C, Kahn CR. Tissue-Specific Insulin Signaling, Metabolic Syndrome, and cardiovascular disease. Arterioscler Thromb Vasc Biol. 2012;32: 2050-2059.

Ribeiro IMR, Ferreira-Neto HC, Antunes, VR. Subdiaphragmatic vagus nerve activity and hepatic venous glucose are differentially regulated by the central actions of insulin in Wistar and SHR. Physiol Rep. 2015;3:1-13.

Silverthorn DE. Fisiologia humana: uma abordagem integrada. 5 ed. Porto Alegre: Artmed, 2010. figura 22-11. Mecanismo celular de ação da insulina.

Short KR. Regulation of Glycemic Control by Physical Activity: A Role for Mitochondria? Diabetes. 2013;62:34-35.

Spike 2. On line: Cambrige Eletronic Design. Version 3 [cited 2016 Out 26] Available from: ced.co.uk.

Szabo AJ, Iguchi A, Burlesson PD, Szabo O. Vagotomy or atropine blocks hypoglycemic effect of insulin injected into ventromedial hypothalamic nucleus. Am. J. Physiol. Endocrinol. Metabol. 1983;244:467-471. 
Szabo A, Szabo AJ. Studies on the nature and mode of action of the insulin- sensitive glucoregulator receptor in the central nervous system. Diabetes. 1975;24:328-336.

Verbene JMA, Sabetghadam A, Korim SW. Neural pathways that control the glucose counterregulatory responde. Font in Neurosci. 2014;8:1-12.

Zhang X, Zheng X, Yuan Y. Treatment of insulin resistance: straight from the gut. Drug Discov Today. 2016;0:1-7. 
